\title{
A PURE DIELECTRIC PIFA FOR WLAN APPLICATIONS
}

\author{
David J Ireland ${ }^{(1)}$, Simon P Kingsley ${ }^{(2)}$ and Steven G O'Keefe ${ }^{(1)}$ \\ ${ }^{(1)}$ Centre for Wireless Monitoring and Applications \\ Griffith School of Engineering \\ Griffith University, Nathan QLD, Australia 4111 \\ d.ireland@griffith.edu.au \\ s.okeefe@griffith.edu.au \\ (2) Antenova Ltd., \\ Far Field House, Albert Road, \\ Stow-cum-Quy, Cambridge, CB25 9AR, UK \\ simon.kingslev@antenova.com
}

\begin{abstract}
This paper proposes a new type of antenna for receiving WLAN signals. The antenna is similar in structural design to a conventional PIFA but comprises a pure dielectric as the main radiating device. It is conjectured here that this novel antenna provides electromagnetic radiation through displacement currents and yet is quite different to the well-known Dielectric Resonator Antenna. This paper will describe this Pure Dielectric PIFA (PD-PIFA) and provide simulation and measurement results supporting the previous conjecture. The salient feature of this antenna was found to be its ability to resist detuning when biologically loaded; a $1.25 \%$ change in resonant frequency was measured when the antenna was placed in a phantom hand.
\end{abstract}

\section{Introduction}

The earliest use of dielectrics in antenna technology is the dielectrically loaded antenna where a conventional conductor is used as the radiator but is modified by the presence of a surrounding external dielectric material. Dielectric loading confers the advantage of good resistance to detuning but has the disadvantage of reducing bandwidth. Some antennas, such as open waveguides, can be filled with an internal dielectric, as in the polyrod, which may be regarded as a degenerate or rudimentary form of lens antenna [1].

In 1939 Robert Richtmyer, a mathematical physicist who worked at both Stanford University and, during the war, at Los Alamos, published a remarkable theoretical paper [2] in which he reasoned that a suitably shaped dielectric object can act as a resonant RF radiator. He offered proof that such a device must radiate based on the boundary conditions at the interface between the dielectric and the surrounding air. This work was not followed up straight away, perhaps because the war intervened, but eventually dielectric resonator antennas (DRAs) were developed in the 1980's and 1990's [3], [4]. DRAs are true dielectric antennas in the sense that it is the dielectric that radiates, rather than any associated metal in the feed system or the ground plane.

DRAs have some excellent properties such as being efficient and having good resistance to proximity detuning [4]. However, they tend to have limited bandwidth, typically around 5\%, whereas with modern communications systems a bandwidth nearer $12-15 \%$ is required. The bandwidth of DRAs can be improved dramatically if the groundplane is taken away from underneath them [5] but this diminishes the advantages and is not popular with the manufacturers of mobile devices, who like to make use of every square millimetre of PCB that is available.

In 2005 a new type of antenna technology was developed that is based on substantially purely dielectric materials and yet which is believed to be different from both (DRAs) and electrically conductive antennas [6], [7]. Electrically conductive antennas such as dipoles can be almost infinitely thin if 
conductivity is sufficiently good, whereas purely dielectric antennas need a finite cross-section to radiate effectively. Pure dielectric antennas are unlike DRAs as the latter are entirely volume devices that radiate like a cavity and if the frequency is halved the device must be correspondingly increased in volume 8 fold. For pure dielectric antennas the resonant frequency is much more dependent on the length of the antenna, as with conventional conductive antennas. It can be shown by simulation that no metal conductor is necessary for a purely dielectric antenna to radiate; however it is required in practice so a feed network may be connected to the antenna for testing. Agreement between simulations and laboratory measurements is good, thus indicating that the technology is real and not a simulation or measurement artefact.

\section{The Purely Dielectric PIFA}

PIFAs are an attractive antenna element to use for small and mobile platforms because of their small size and their ability to work in very close proximity to a groundplane. Here we examine the performance of a purely dielectric PIFA. Figure 1 shows the proposed PD-PIFA structure where a ceramic material with a relative permittivity of 93 was used. Similar to the conventional PIFA, one end of the ceramic material is short circuited to the ground plane while the feed is placed further along the material in order to provide a suitable impedance match. Several particular favourable attributes of this structure are that, the feed pin merely rests on the dielectric rather then being probe fed, the ceramic is located only $2 \mathrm{~mm}$ above the ground-plane and yet will be shown to provide an efficient electromagnetic radiation mechanism. Achieving such proximity to the ground-plane is difficult using conventional conductive antennas because of the significant coupling of electromagnetic energy between the groundplane and radiating element.

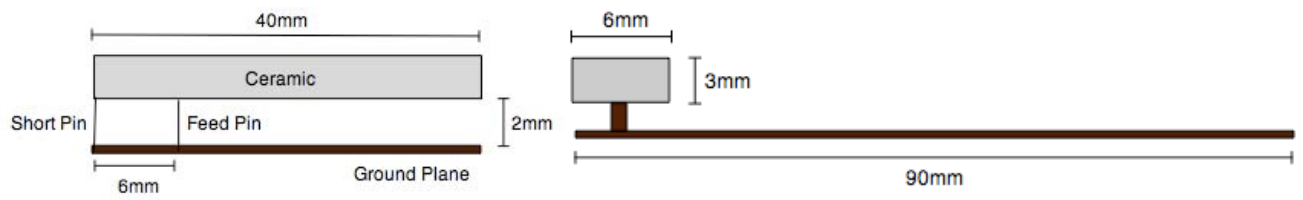

Fig. 1 Front and Side Views respectively of the PD-PIFA

Figure 2(a) shows the magnitude of the electric field inside the ceramic material showing the field diminishing as it approaches the open end of the ceramic. This resembles that of a conductive antenna, which further suggests the radiation mechanism is somewhat different to the previously mentioned conventional dielectric antennas and more akin to a traditional monopole configuration.

A well-known aspect of dielectric resonator antennas is that a significant portion of the electric field is contained within the material itself. This tends to decrease the susceptibility of the antenna to detuning by biological loading. This has been explored by examining the radiation performance in free space and when the antenna has been loaded by a phantom hand, which has similar electrical and mechanical properties to a human hand. This was done in order to ascertain whether the PD-PIFA will retain the ability of remaining tuned when proximity loaded, a property the DRA is well known for. Figure 2(b) shows the phantom hand and the PD-PIFA that was used for analysis of the reflection coefficient and far-field properties.

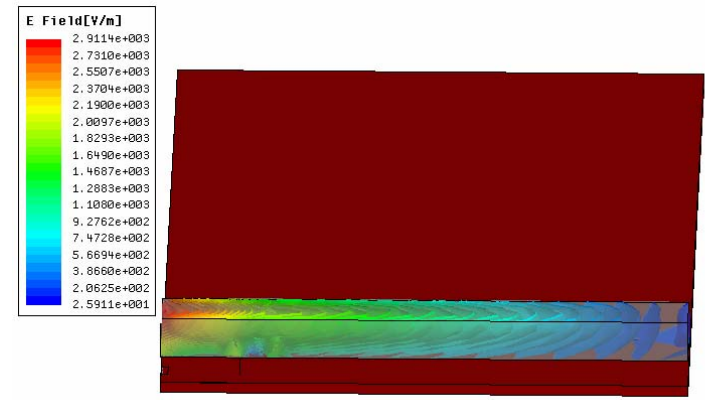

(a)

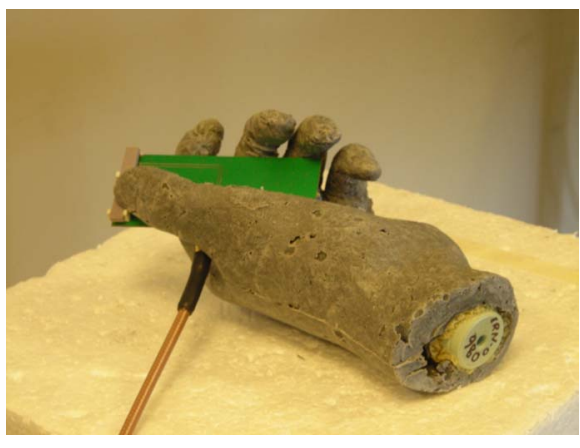

(b)

Fig. 2 (a) Simulated Electric Field inside the dielectric (b) PD-PIFA mounted in the phantom hand 


\section{Performance}

Figure 3(a) shows the measured and simulated return loss for the PD-PIFA revealing a high consistency between simulation and measured results. The measured ( $-10 \mathrm{~dB}$ return loss) bandwidth is shown to be $90 \mathrm{MHz}$ or $3.8 \%$, which is adequate for communication over a LAN. Also included in this figure is the simulated return loss when a simple two-component matching circuit has been included, resulting in a larger impedance bandwidth of $147 \mathrm{MHz}$ or $6.1 \%$. An additional trace is given showing the impact of detuning due to the phantom hand. Remarkably, minimal impact was observed, with the resonant frequency shifting by only $30 \mathrm{MHz}$ or $1.25 \%$.

The measured radiation efficiency with and without the phantom hand is given in figure 3(b). When the antenna was located in free space the average radiation efficiency was found to be $69 \%$ at the resonant bandwidth $(2400-2500 \mathrm{MHz})$ while the average efficiency from $2300-2500 \mathrm{MHz}$ was $56 \%$, both high values for such proximity to the ground-plane. When the phantom hand was included in the measurement however, a significant drop of efficiency was recorded; the absorption by the phantom caused, on average, a drop in efficiency of approximately $4.4 \mathrm{~dB}$ at the resonant bandwidth. This value is typical however for most antenna configurations.

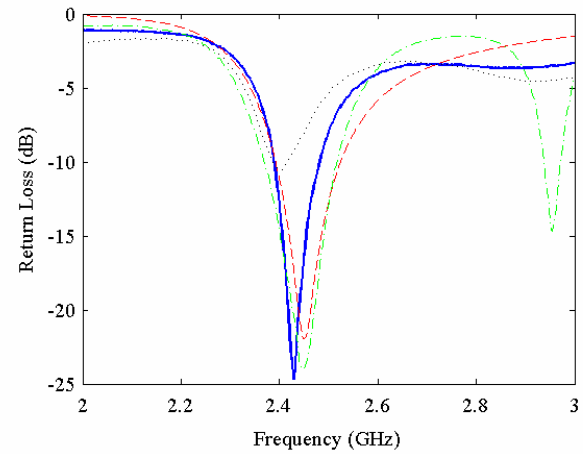

(a)

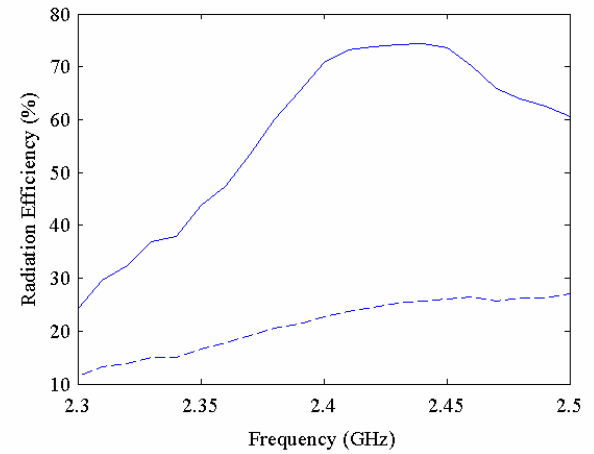

(b)

Fig. 3 (a) Return Loss, Measured (-); simulated (--); matching Circuit (-.); phantom hand (.) (b) Measured Radiation Efficiency, free space (-); with phantom hand (--)

Figures 4(a) and 4(b) show the measured far-field radiation patterns respectively with and without the phantom hand respectively. The distortion of the far-field radiation pattern when the phantom hand was included, reflects the hindrance biologically loading has made and yet adds evidence of the ability of the proposed antenna structure to resist detuning.

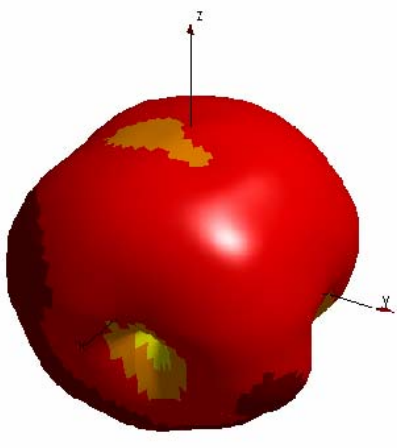

(a)
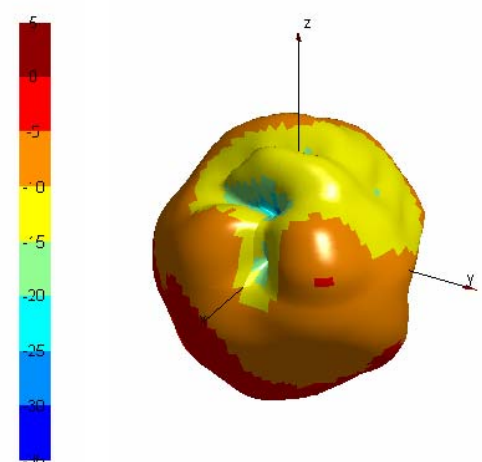

(b)

Fig. 4 (a) Measured Far-Field Radiation Pattern (dBi), free space, (b) Phantom Hand 


\section{Conclusion}

This paper has proposed, what is believed to be, a novel antenna where electromagnetic radiation is achieved through displacement currents rather then conductive currents, and yet the antenna shares similarities in terms of field distribution to an antenna featuring a conductive radiating element. Empirical evidence was shown to support this hypothesis. Furthermore, measurement evidence was shown that this antenna may share favourable qualities from both conventional conductive and dielectric resonator antennas in terms of propinquity to the ground-plane, resistance to detuning and high radiation efficiency. Although not small by today's standards for a $2.4 \mathrm{GHz}$ antenna, it is believed such an antenna may potentially provide a competitive commercial product for applications where detuning is a critical issue.

Future work will be taken in several directions; firstly, a thorough optimisation of the antenna structure will be implemented in order to establish the numerical trade-off between the proximity to the groundplane, size of the dielectric material and the electromagnetic radiation performance. Secondly, research will be carried out on the viability of establishing multiple resonant bandwidths by inserting conductive elements on the surface of the dielectric material.

\section{Acknowledgements}

The authors wish to thank Scott Tyler for his diligent help in making the prototypes.

\section{References}

[1] G. Wilkes, "Wavelength lens", Proc. IRE, 206-212, 1948.

[2] R.D. Richtmyer "Dielectric resonators," J. Applied Physics, 10, pp 391 - 398, 1939.

[3] S.A. Long, M.W. McAllister and L.C. Shen, "The Resonant Cylindrical Dielectric Cavity Antenna," IEEE Transactions on Antennas and Propagation, AP-31, pp 406-412, 1983.

[4] R.K. Mongia and P. Bhartia, "Dielectric Resonator Antennas - A Review and General Design Relations for Resonant Frequency and Bandwidth," International Journal of Microwave and Millimetre-Wave Computer-Aided Engineering, 4, (3), pp 230-247, 1994.

[5] Z. Wang, C. C. Chiau, X. Chen, B. S. Collins, S. P. Kingsley and S. Puckey, "Study and Optimisation of A Broadband Dielectric Antenna," presented at the IEEE International Workshop on Antenna Technology: Small Antennas and Novel Metamaterials, Singapore, March 7 - 9, 2005.

[6] J.M Ide, S.P Kingsley, S.G O'Keefe, S.A Saario "A novel wide band antenna for WLAN applications", presented at the 2005 IEEE AP-S International symposium \& USNC/URSI National Radio Science meeting, 3-8 July 2005, Washington, DC.

[7] J M Ide, S P Kingsley, S G O'Keefe and S Saario, "Pure dielectric antennas and related devices", UK Patent No 2422248. 